\title{
From Flax Fibers to Activated Carbon Electrodes: The Role of Fiber Refining
}

\author{
Hongwei Li, ${ }^{\text {a,b }}$ Yucheng Feng, ${ }^{\text {a,b }}$ Lvqiao Tang, ${ }^{\text {a,b }}$ and Fei Yang ${ }^{\text {a,b,* }}$ \\ Flax-based activated porous carbon materials (APCs) were prepared via \\ $\mathrm{KOH}$ and urea synergistic activation in the carbonization process using \\ flax pulp as a biocompatible and eco-friendly biomass precursor. A refining \\ process was used to pretreat the flax pulp fibers, which has been known \\ to improve and optimize the performance of APCs. The morphological and \\ physicochemical structures of APCs were investigated, and the results \\ showed that APCs exhibited high specific surface area and porous \\ microstructure. Furthermore, APCs were rationally designed as a \\ sustainable electrode material. The APC prepared by $60^{\circ} \mathrm{SR}$ (Shopper- \\ Riegler beating degree) flax pulp, named APC-60, exhibited the highest \\ specific capacitance of $265.8 \mathrm{~F} / \mathrm{g}$ at a current density of $0.5 \mathrm{~A} / \mathrm{g}$. The \\ specific capacitance retention at $59 \%$ remained for the APC-60 electrodes \\ at a high current density of $10 \mathrm{~A} / \mathrm{g}$. These results suggested that the flax- \\ based APCs could be a promising carbon-based electrode material for \\ sustainable electrochemical energy storage.
}

Keywords: Flax pulp; Refining process; Activated porous carbon; Electrochemical performance

Contact information: a: State Key Laboratory of Pulp and Paper Engineering, South China University of Technology, Guangzhou 510640, China; b: United Lab of Plant Resources Chemistry and Chemical Engineering, South China University of Technology, Guangzhou 510640, China;

*Corresponding author: yangfei@scut.edu.cn

\section{INTRODUCTION}

In recent years, with the rapid development of industrialization and a large increase in population, exhaustible resources, such as fossil energy, are on the verge of depletion (Hadi et al. 2015). Resources and energy issues have become common topics of human development in the $21^{\text {st }}$ century, forcing society to start looking for alternative energy sources, more advanced and sustainable energy storage, and conversion technologies (Liang et al. 2013).

The supercapacitor is a new type of energy storage device that exhibits the advantages of high-power density, rapid charge-discharge rates, and low cost, making it likely the best choice for future energy storage equipment (Yu et al. 2015; Borenstein et al. 2017). The material of the electrode, one of the most important components of a supercapacitor, affects the capacitive performance of the electrochemical capacitor in terms of energy and power density (Mohammed et al. 2019). Biomass-based activated porous carbon materials show great potential in energy storage and conversion applications due to many excellent characteristics, such as abundant natural sources, environmental friendliness, a porous network structure, a high specific surface area, tunable porosities, and relatively low density (Zhang and Zhao 2009). Recently, biomass-based activated porous carbon has been a focus of attention, and the use of various biomass resources as 
raw materials for the activated porous carbon materials can be applied to supercapacitor electrode materials (Tan et al. 2017; Yang et al. 2018; Wu et al. 2019).

The preparation of activated carbons can be achieved by two main methods, namely, physical activation (Sui et al. 2014) and chemical activation (Yang and Qiu 2010). Most of the biomass-based activated carbon materials are prepared via a chemical activation method (Deng et al. 2010; Wang et al. 2015; Ma et al. 2019), which is an efficient method that mixes the activator and the precursor with high-temperature carbonization under a protective gas to obtain a carbon material with a layered porous structure. During the activation process, a series of chemical reactions are carried out mainly by the activator and the precursor, and the carbon skeleton is consumed to make pores. At present, commonly used activators are: $\mathrm{KOH}$ (Wang and Kaskel 2012), $\mathrm{NaOH}$ (Islam et al. 2017), $\mathrm{K}_{2} \mathrm{CO}_{3}$ (Li et al. 2017), $\mathrm{ZnCl}_{2}$ (Yu et al. 2017), and $\mathrm{H}_{3} \mathrm{PO}_{4}$ (Girgis et al. 2007). $\mathrm{KOH}$ is the most widely used and effective among many kinds of activators. In addition, one study (Chen et al. 2017) has shown that the addition of urea in the carbonization process not only introduces a certain amount of $\mathrm{N}$ element into the carbon material, but it also plays a role in pore formation. This is attributed to the gases, such as $\mathrm{CO}_{2}, \mathrm{NH}_{3}$, and water vapor, generated by urea pyrolysis. These gases play the role of poreforming when they escape from the inside of the carbon material. Additionally, the method of adding the activator also greatly affects the activation of the carbon material. The traditional method mixes the carbon precursor and the activator in a certain proportion in a solid state, resulting in uneven mixing of the activator and the carbon material, inadequate contact, and low pore-forming efficiency.

Flax, an annual herb, has wide distribution around the world. Flax and its ancillary products are widely used in chemical, food, and medical fields (Dhakal et al. 2013; Yan et al. 2014). Flax fibers are favored in the textile and paper fields due to their renewability, biocompatibility, high tensile strength, fast swelling rate, and high expansion coefficient (Sain and Fortier 2002; Martin et al. 2016). For example, adding flax pulp to the preparation of cigarette paper has been widely used (Shen et al. 2014). In addition, flax used as a carbon precursor to prepare activated porous carbon has also, in recent years, exhibited great potential due to the above-mentioned advantages (Wu et al. 2017; Tang et al. 2019; He et al. 2020).

However, the application of flax fiber to carbon materials in previous studies still has some problems. Few studies have paid attention to the pretreatment process of the precursors. When the activator is formulated as a solution, although the flax fibers exhibit an excellent adsorption property, it is still difficult for activator to enter and disperse evenly in the fibers due to the poor accessibility of the untreated fibers, which directly leads to a low activation effect, resulting in a low electrochemical performance of the prepared carbonized materials. Refining, a commonly used process in papermaking (Marrakchi et al. 2011; Mao et al. 2019), improves the physical properties of pulp fibers, including their structure and size, and further improves their specific surface area and swelling performance (Chen et al. 2016; Motamedian et al. 2019). During refining the wet cellulosic fibers are repeatedly compressed and sheared, resulting in internal delamination of the cell walls, and increased swelling in the wet state. Thus, the use of the refining process to promote fibrillation of the flax pulp fibers further enhances the flax pulp fibers' ability to absorb the activator and increases the effective activation area of the activated carbon materials during the carbonization process. Refining causes the fibers to fibrillate and increases of the accessibility of fibers for activator, which further promotes the fibers' swelling and enhances their liquid absorption performance, making the activator more 
easily enter the fiber cell cavity (Gharehkhani et al. 2015). Additionally, refining is a mature process in papermaking, and the beating degree of fibers is easier to control. Therefore, the refining treatment of flax pulp fibers improves the activator's pore-forming efficiency. It is thus of great research importance to improve the structural properties and electrochemical properties of carbon materials by adjusting the beating degree.

In this study, flax pulp fibers with different beating degrees of 40,60, and 80 degrees Schopper-Riegler ( $\left.{ }^{\circ} \mathrm{SR}\right)$ were used as the bio-based starting material for preparing activated carbon. The physicochemical properties and structural characteristics of the activated porous carbon materials (APCs) were investigated by scanning electron microscopy (SEM), $\mathrm{N}_{2}$ isothermal adsorption-desorption test, X-ray diffraction (XRD), and Raman spectra. In order to study the electrochemical performance of APCs, the electrode materials derived from flax-based activated carbon were then fabricated. Tests measuring cyclic voltammetry $(\mathrm{CV})$, galvanostatic charge-discharge (GCD), and electrochemical impedance spectroscopy (EIS) were conducted by a CHI760E electrochemical workstation. This study provides the possibility to improve the electrochemical performance of as-prepared APCs with refining process. And it also provides a direction for other biomass raw materials to get efficiently utilization in activated carbon materials.

\section{EXPERIMENTAL}

\section{Materials}

Bleached flax pulp was purchased from Fengyuan Special Paper Co., Ltd. (Xingtai, China). In order to ensure the formation uniformity of the paper, this kind of bleached flax market pulp board used for cigarette paper and other tissue paper has been cut off before leaving the factory, so the beating degree was relatively high and nearly $40{ }^{\circ} \mathrm{SR}$. The pulp fibers were refined in a Mark VI type PFI refiner (Hamjern Maskjn, Hamar, Norway) to beating degrees of $40{ }^{\circ} \mathrm{SR}, 60{ }^{\circ} \mathrm{SR}$, and $80{ }^{\circ} \mathrm{SR}$, respectively. The beating pressure was $(3.33 \pm 0.1) \mathrm{N} / \mathrm{mm}$. The rated speed of the beater roll was $(1460 \pm 30) \mathrm{r} / \mathrm{min}$. And the line speed difference between beater roll and beater housing was $(6.0 \pm 0.2) \mathrm{m} / \mathrm{s}$. Potassium hydroxide $(\mathrm{KOH})$ and urea were supplied by Guangdong Guanghua Sci-Tech Co., Ltd. (Guangzhou, China). Hydrochloric acid $(\mathrm{HCl})$ was purchased from Guangzhou Chemical Reagent Factory (Guangzhou, China). Polytetrafluoroethylene (PTFE), 60 wt\% concentrated dispersion, was purchased by Aladdin (Shanghai, China). All chemical reagents used in this study were of analytical grade without further purification.

\section{Methods}

Preparation of flax-based activated porous carbon

A graphical illustration of flax-based activated porous carbon formation process is shown in Fig. 1. The flax pulp fibers with different beating degrees were mixed with potassium hydroxide and urea in a mass ratio of 1:3:1 (based on dry pulp) and immersed in a certain amount of deionized water to make the pulp consistency reached $5 \mathrm{wt}$.\%. The mixtures were stirred constantly and intensively. Then, flax pulp fibers containing $\mathrm{KOH}$ and urea were frozen in a low temperature refrigerator at $-20{ }^{\circ} \mathrm{C}$ for $24 \mathrm{~h}$, and subsequently the flax-based carbon precursors containing $\mathrm{KOH}$ and urea were obtained via freeze-drying in a lyophilizer for $48 \mathrm{~h}$ at $-58{ }^{\circ} \mathrm{C}$ and $22 \mathrm{~Pa}$. 
The flax-based carbon precursors with different beating degrees were put into a vacuum tube furnace (OTF-1200X-III; Hefei Kejing Materials Technology Co., Ltd., Hefei, China), and then a stable nitrogen flow of $100 \mathrm{~cm}^{3} / \mathrm{min}$ was passed after vacuum. During the carbonization process, the temperature was first increased to $200{ }^{\circ} \mathrm{C}$ for $2 \mathrm{~h}$ at a rate of $5{ }^{\circ} \mathrm{C} / \mathrm{min}$. Next, the temperature was increased to $800{ }^{\circ} \mathrm{C}$ for $2 \mathrm{~h}$ at a rate of 3 ${ }^{\circ} \mathrm{C} / \mathrm{min}$ to obtain the flax-based APCs with different beating degrees. After the temperature cooled to room temperature, the as-prepared samples were washed with a $0.5 \mathrm{M}$ solution of hydrochloric acid to wash and clean the alkali on the surface of carbon materials, so that the pore structure can be exposed. Then as-prepared carbon materials were washed to neutrality with distilled water to remove the agent residues and other impurities. Afterward, the flax-based activated porous carbon was dried in oven at $105^{\circ} \mathrm{C}$ and stored for further analysis and characterization. The as-prepared flax-based activated porous carbons with different beating degrees were named APC-40, APC-60, and APC-80.

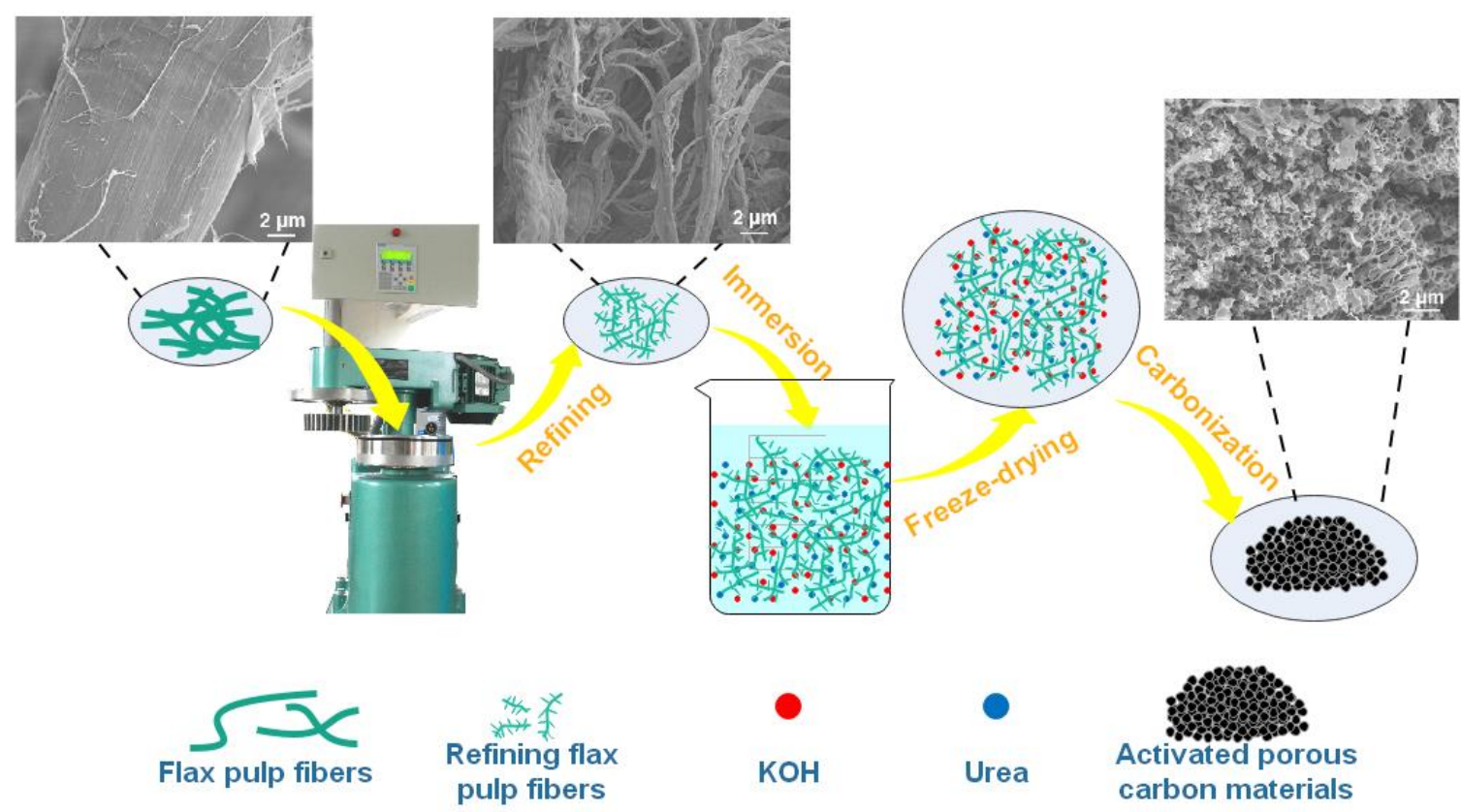

Fig. 1. Schematic diagram of the flax-based activated porous carbon preparation process

\section{Characterization}

The morphologies of APCs were characterized using a field-emission scanning electron microscope (FE-SEM Merlin; Micromeritics instrument Ltd., Oberkochen, Germany). The phases of the as-prepared APCs were analyzed by an X-ray diffractometer (D8-advance; Bruker, Karlsruhe, Germany). The scanning speed was $2 \% \mathrm{~min}$ and the scanning angle was from $10^{\circ}$ to $90^{\circ}$. Raman spectra were carried out on a Raman spectrometer (LabRAM Aramis, H.J.Y, Paris, France). The elemental contents of the asprepared samples were carried out on an elemental analyzer (Vario EL cube, Elementar, Hanau, Germany), and a multifunctional X-ray photoelectron spectroscopy (Axis Ultra DLD, Kratos, Manchester, England) with a monochromatic X-ray source of Al Ka (1486.6 $\mathrm{eV})$.

An automatic physisorption analyzer (ASAP-2460; Micromeritics, Norcross, Georgia, USA) was used to characterize the specific surface area and pore size distribution of APCs. The $\mathrm{N}_{2}$ adsorption-desorption test was carried out at $-196{ }^{\circ} \mathrm{C}$. Before testing, the 
as-prepared samples were degassed under vacuum at $150{ }^{\circ} \mathrm{C}$ for $12 \mathrm{~h}$ to remove impurities and residual moisture on the surface. According to the experimental data, the BrunauerEmmett-Teller (BET) method was used to calculate the specific surface area, and the Density Functional Theory (DFT) model was used to calculate the pore size distribution of the samples.

\section{Electrochemical measurements}

Electrochemical properties of APCs were characterized using an electrochemical workstation (CHI760E; CH Instruments; Shanghai, China) in $1 \mathrm{M} \mathrm{H}_{2} \mathrm{SO}_{4}$ aqueous solution. The CV, GCD, and EIS were performed in the three-electrode system at room temperature. For preparing the testing electrode, $4 \mathrm{mg}$ of porous carbon samples and $15 \mu \mathrm{L}$ of PTFE were dispersed in $1 \mathrm{~mL}$ mixed solution of $\mathrm{H}_{2} \mathrm{O}$ and ethanol $(3: 2$, in volume). The above mixture was sonicated for $30 \mathrm{~min}$ to be dispersed completely, and $5 \mu \mathrm{L}$ of the dispersed mixture was coated onto a glassy carbon electrode. This was dried at room temperature to obtain the testing electrode, which was used as the working electrode, in a three-electrode mode. The Pt electrode and the $\mathrm{Ag} / \mathrm{AgCl}$ electrode served as the auxiliary electrode and the reference electrode, respectively. The $\mathrm{CV}$ tests were investigated at different scan rates $(5,10,20,50,100$, and $200 \mathrm{mV} / \mathrm{s})$ in the potential window of -0.2 to $0.8 \mathrm{~V}$. The specific capacitance of the electrode at different scan rates can be calculated by the following formula,

$$
C=\int(I d V) /(v m \Delta V)
$$

where $I$ is the test actual current $(\mathrm{A}), v$ is the scan rate $(\mathrm{V} / \mathrm{s}), m$ is the actual mass of the active material $(\mathrm{g})$, and $\Delta V$ is the potential window $(\mathrm{V})$.

The EIS tests were investigated in the frequency range of $0.01 \mathrm{~Hz}$ to $100 \mathrm{kHz}$ with an alternating current amplitude of $5 \mathrm{mV}$. The GCD tests were performed at different current densities $(0.5,1,2,5$, and $10 \mathrm{~A} / \mathrm{g})$ in the potential window of -0.2 to $0.8 \mathrm{~V}$. The specific capacitance of the as-prepared working electrode was calculated from the GCD curves by using Eq. 2,

$$
C=(I \Delta t) /(m \Delta V)
$$

where $I(\mathrm{~A})$ is the constant current, $\Delta t(\mathrm{~s})$ is the discharge time, $m(\mathrm{~g})$ is the mass of electrode material, and $\Delta V(\mathrm{~V})$ is the voltage change during discharge.

\section{RESULTS AND DISCUSSION}

\section{Morphological Analysis}

The surface morphology and the pore structures of the APCs and of the flax pulp fibers with different beating degrees were investigated via field-emission scanning electron microscopy. Figure 2 shows the SEM images of flax pulp fibers with different beating degrees of 40, 60, and $80^{\circ} \mathrm{SR}$ and the as-prepared samples APC-40, APC-60, and APC80. It can be seen from Figs. 2 a through $2 \mathrm{c}$ that, with the increase of beating degree, the flax pulp fibers were gradually broken and fibrillated, and the fiber surface was divided into filaments, helping the flax pulp fibers to absorb the activator and increase the effective contact area between the activator and the fibers. This eventually led to an improved poreforming efficiency during the high-temperature activation process. Furthermore, Figs. 2d through $2 \mathrm{f}$ clearly indicates that the flax-based carbon materials, after a high-temperature 
activation at $800{ }^{\circ} \mathrm{C}$, showed a porous network microstructure. In addition, the surface pore structures of APC-40, APC-60, and APC-80 were notably different, which can be attributed to the refining treatment of the flax pulp fibers. For the APCs, the above SEM images (Figs. $2 \mathrm{~d}$ through $2 \mathrm{f}$ ) confirmed that with the increase of the beating degree of fibers, the surface of porous carbon materials tended to become fragmented with a porous structure.

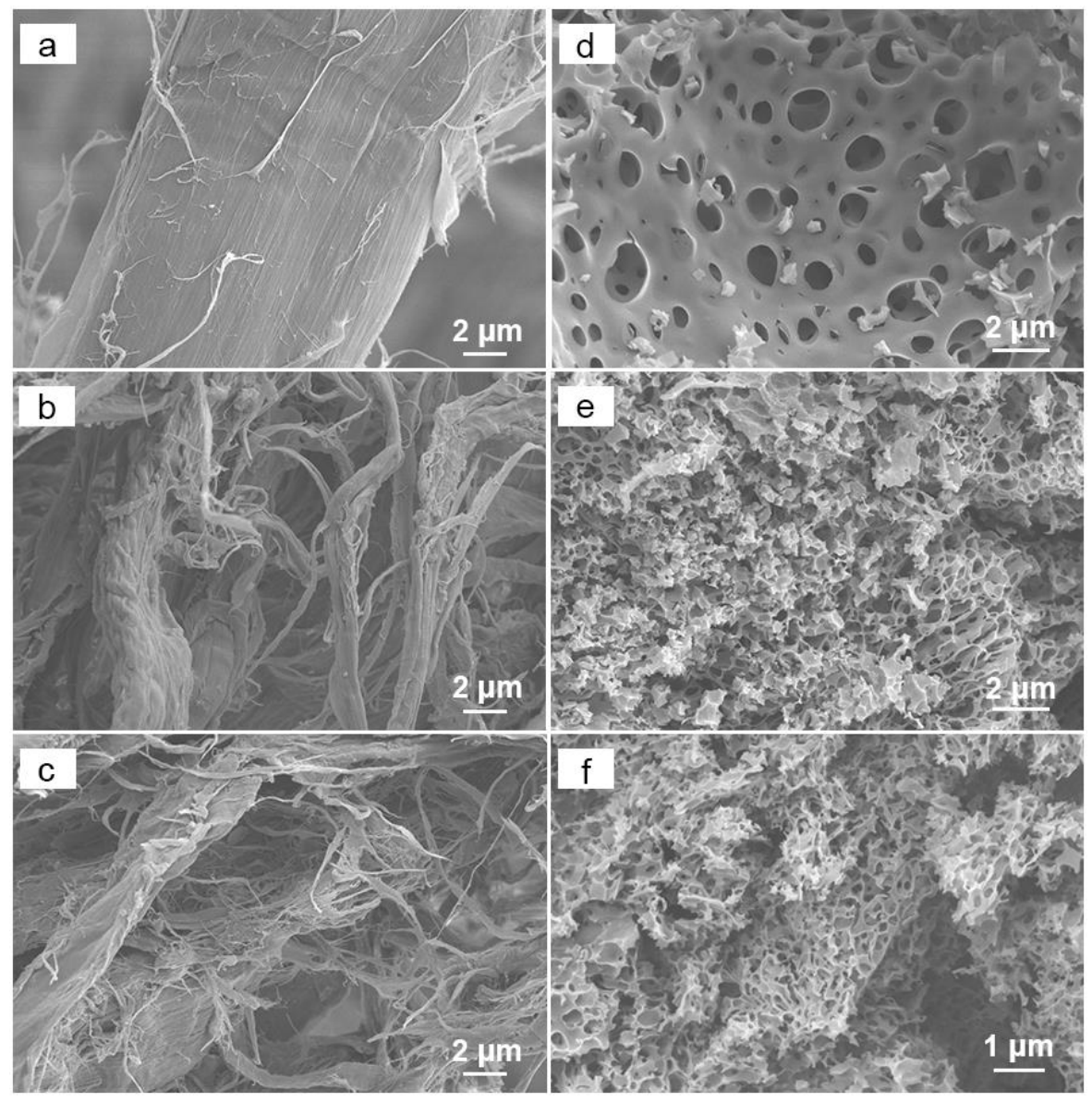

Fig. 2. SEM images of flax pulp fibers with beating degrees of: (a) $40^{\circ} \mathrm{SR}$, (b) $60^{\circ} \mathrm{SR}$, and (c) 80 'SR, and the as-prepared samples (d) APC-40, (e) APC-60, and (f) APC-80

\section{Specific Surface Area and Pore Size Distribution}

The specific surface area and pore size distribution of the flax-based activated porous carbon materials were tested by a four-station automatic specific surface area and porosity analyzer. Figures $3 \mathrm{a}$ and $3 \mathrm{~b}$ show the $\mathrm{N}_{2}$ adsorption-desorption isotherm and the pore size distribution curves of APC-40, APC-60, and APC-80. According to the classification of the International Union of the Pure and Applied Chemistry (IUPAC), the flax-based APCs show type I (b) adsorption isotherm characteristics. This type of adsorption isotherm exhibits a small hysteresis loop, which is mainly dominated by micropores. As is shown in Fig. 3a, when the relative pressure $\left(P / P_{0}\right)$ was extremely low, the quantity adsorbed rose sharply, which was attributed to the interaction between the sorbent and the adsorbate in micropores markedly enhanced, causing the micropores to fill at extremely low relative pressures. When the saturation pressure was reached $\left(P / P_{0}>\right.$ 0.99), the adsorbates aggregated and the curve slightly rose. Additionally, the type I (b) adsorption isotherm explained the wide pore size distribution of micropores, and the 
presence of narrower mesopores. Figure $3 \mathrm{~b}$ also demonstrates that the pore structures of APCs included a large number of mesopores, with a small amount of mesopores (pore width less than $2.5 \mathrm{~nm}$ ).

The BET surface areas of APCs, displayed in Table 1, clearly demonstrated that the APCs activated by synergistic activation of $\mathrm{KOH}$ and urea exhibited high specific surface areas and porous structures. Moreover, with the increase of the beating degree from 40 to $80{ }^{\circ} \mathrm{SR}$, the specific surface area increased from 957.7 to $1255.3 \mathrm{~m}^{2} / \mathrm{g}$, and the pore volume rose from 0.5251 to $0.7245 \mathrm{~cm}^{3} / \mathrm{g}$, respectively. These increases mainly resulted because, as the beating degree increased, the swelling properties of flax pulp fibers also grew, more $\mathrm{KOH}$ and urea were distributed on the fiber surface and in the cell cavity, and the reaction between flax pulp fibers and activator strengthened during the high temperature activation process, causing higher pore-forming efficiency. In addition, as the beating degree increased, the average pore diameter became slightly wider due to the enhanced activation and the opening of channels between adjacent micropores. Finally, the multiple micropores were superimposed to form mesopores with larger pore diameters. Additionally, the high specific surface area and porosity increased the size of the contact site between the electrolyte ions and the surface of the carbon materials, enhanced the charge transfer, and increased the specific capacitance of the supercapacitor.
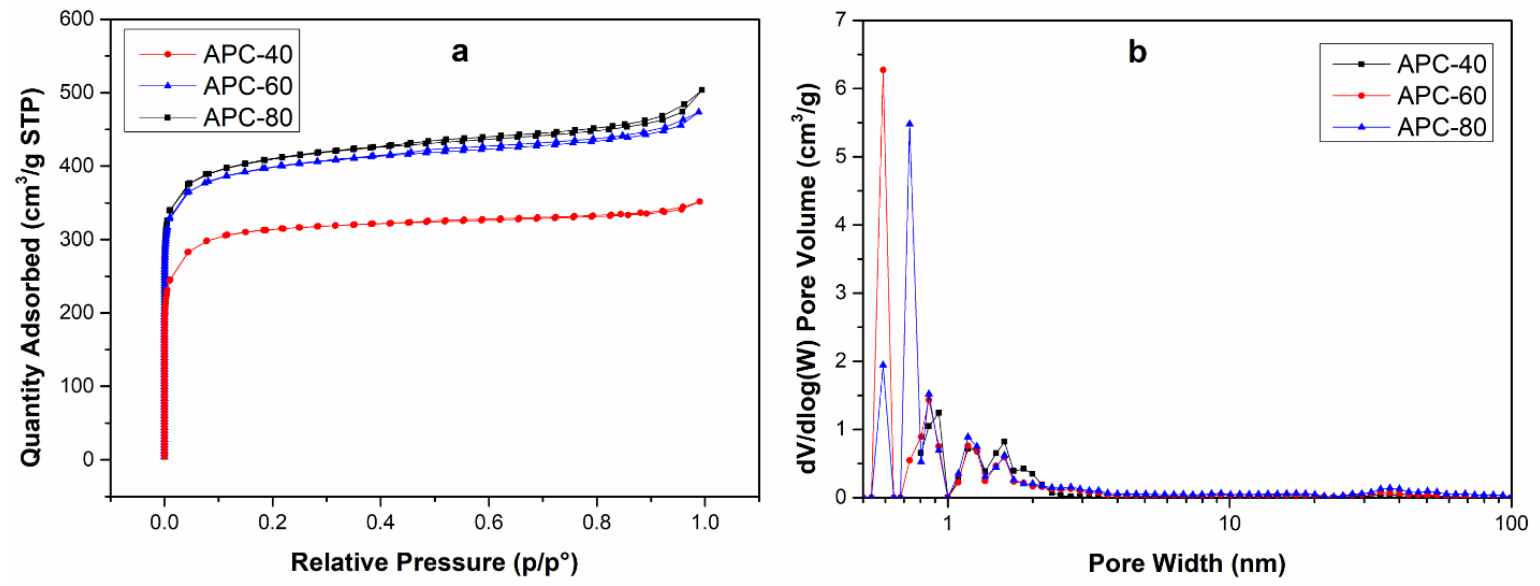

Fig. 3. (a) $\mathrm{N}_{2}$ adsorption-desorption isotherms, and (b) pore size distributions, calculated by the DFT method of APC-40, APC-60, and APC-80

Table 1. BET Measurement of APC-40, APC-60, and APC-80

\begin{tabular}{|c|c|c|c|c|c|}
\hline Code & $\begin{array}{c}S_{\text {BET }} \\
\left(\mathrm{m}^{2} / \mathrm{g}\right)\end{array}$ & $\begin{array}{c}S_{\text {mic }} \\
\left(\mathrm{m}^{2} / \mathrm{g}\right)\end{array}$ & $\begin{array}{c}V_{\top} \\
\left(\mathrm{cm}^{3} / \mathrm{g}\right)\end{array}$ & $\begin{array}{c}V_{\text {mic }} \\
\left(\mathrm{cm}^{3} / \mathrm{g}\right)\end{array}$ & $D_{\mathrm{P}}$ \\
\hline APC-40 & 957.7 & 793.4 & 0.5251 & 0.4122 & 2.193 \\
\hline APC-60 & 1226.0 & 955.6 & 0.7008 & 0.4965 & 2.287 \\
\hline APC-80 & 1255.3 & 941.8 & 0.7245 & 0.4946 & 2.308 \\
\hline
\end{tabular}

Table 2. Elemental Contents of APC-40, APC-60, and APC-80

\begin{tabular}{|c|c|c|c|c|c|c|}
\hline Code & $\begin{array}{c}\text { C1s } \\
(\%)\end{array}$ & $\begin{array}{c}\text { O1s } \\
(\%)\end{array}$ & $\begin{array}{c}\text { N1s } \\
(\%)\end{array}$ & $\begin{array}{c}\text { C } \\
(\%)\end{array}$ & $\begin{array}{c}\mathrm{N} \\
(\%)\end{array}$ & $\begin{array}{c}\mathrm{H} \\
(\%)\end{array}$ \\
\hline APC-40 & 90.38 & 9.19 & 0.43 & 48.61 & 0.69 & 4.77 \\
\hline APC-60 & 86.12 & 13.16 & 0.73 & 65.69 & 0.52 & 4.16 \\
\hline APC-80 & 81.32 & 17.53 & 1.15 & 74.32 & 1.05 & 4.54 \\
\hline
\end{tabular}




\section{Chemical Surface and Structural Characterization}

The elemental contents of APCs, shown in Table 2, illustrated that APCs were mainly composed of $\mathrm{C}$, which was doped with relatively high $\mathrm{O}$ amounts and small amounts of N. And the results of elemental analysis and XPS analysis were roughly the same. As the beating degree increased, the O1s on the surface of the activated porous carbon materials increased from $9.19 \%$ to $17.53 \%$, and the N1s increased from $0.43 \%$ to $1.15 \%$. These increases resulted because an increase of beating degree enhances the swelling properties of the flax pulp fibers, and more $\mathrm{KOH}$ and urea participate in the effective activation reaction. Thereby, more $\mathrm{N}$ and $\mathrm{O}$ elements were introduced, which enhanced the surface reaction activity, increased the wettability to the electrolyte, and improved the electrochemical performance of APCs.

The structural characterization of the as-prepared flax-based activated porous carbon materials were further confirmed by Raman spectroscopy and X-ray diffraction. Figure 4a shows the as-prepared APC-40 having two broad peaks located at $1340 \mathrm{~cm}^{-1}$ and $1596 \mathrm{~cm}^{-1}$, which correspond to the $\mathrm{D}$ band and the $\mathrm{G}$ band, respectively. The $\mathrm{D}$ band mainly reflected the degree of disorder and the structural defects of the carbon materials. The $\mathrm{G}$ band, caused by the bond extension of the $\mathrm{sp}^{2}$ carbon atom pair, represents the graphite peak, which reflected the degree of graphitization of carbon materials (Daems et al. 2014; Fan et al. 2014). The samples APC-60 and APC-80 both showed that two broad peaks were located at $1340 \mathrm{~cm}^{-1}$ and $1589 \mathrm{~cm}^{-1}$, which can also be matched to the $\mathrm{D}$ band and $\mathrm{G}$ band, respectively. Moreover, the $I_{\mathrm{D}} / I_{\mathrm{G}}$ ratios of the three samples were all close to 1 , with little difference. However, the position of the $\mathrm{G}$ band shifted from $1596 \mathrm{~cm}^{-1}$ to $1589 \mathrm{~cm}^{-1}$, indicating that the activation reaction was enhanced, and the $\mathrm{N}$ element introduced by urea and the pore structures of APCs increased, resulting in defects in the graphite structure.
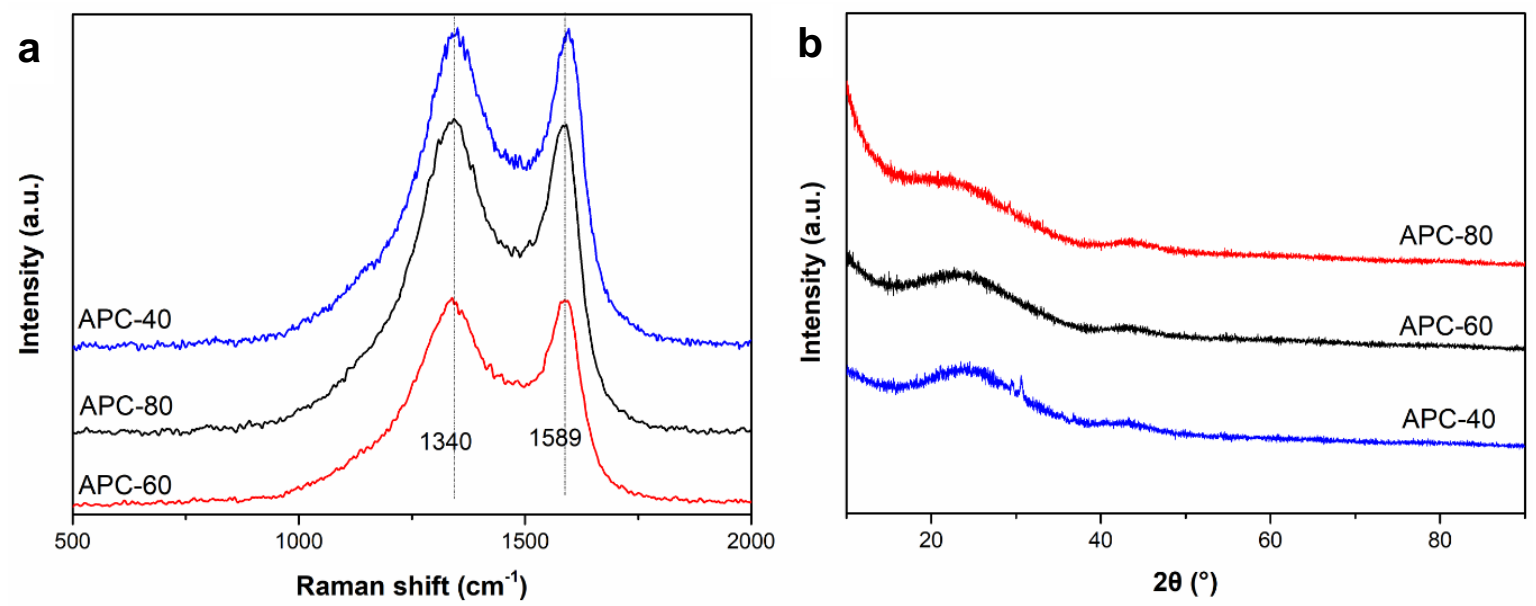

Fig. 4. (a) Raman spectra of APC-40, APC-60, and APC-80; (b) X-ray diffraction patterns of APC40, APC-60, and APC-80

The XRD spectra of APCs are shown in Fig. 4b. All three samples showed a broad diffraction peak at $2 \theta \approx 25^{\circ}$, indicating that the flax-based activated carbon materials belonged to amorphous carbon and exhibited a certain graphitization tendency. Additionally, the intensity of the characteristic diffraction peak at $2 \theta \approx 25^{\circ}$, caused by the graphene (002) plane, displayed the degree of graphitization of the as-prepared APCs. Apparently, as the beating degree increased, the degree of graphitization of APCs 
decreased. This decrease was mainly due to the damage to the flax pulp fibers' crystalline structure by the refining process and the stronger activation reaction, which consumed more carbon atoms of the carbon precursors, severely damaged the carbon skeleton, and reduced the degree of graphitization. The end results was a poor conductivity, affecting the electrochemical performance of APCs.

\section{Chemical Surface and Structural Characterization}

Cyclic voltammetry

Figure 5 shows the CV curves of the as-prepared samples (APC-40, APC-60, and APC-80) profile in the potential window of -0.2 to $0.8 \mathrm{~V}$ at different scan rates. The CV curves display an almost symmetrical rectangle shape, and no distortion occurred even at a high scan rate of $200 \mathrm{mV} / \mathrm{s}$, illustrating that the flax-based APCs had a fast charge transfer ability. This ability was mainly due to the structure of APCs - a hierarchical porous carbon structure with abundant large-size micropores and a large number of narrow mesoporesthat provided ions with a highly accessible surface area, shortened the ion diffusion path at the electrode/electrolyte interface, and thereby promoted the rapid transfer of ions (Feng et al. 2015).
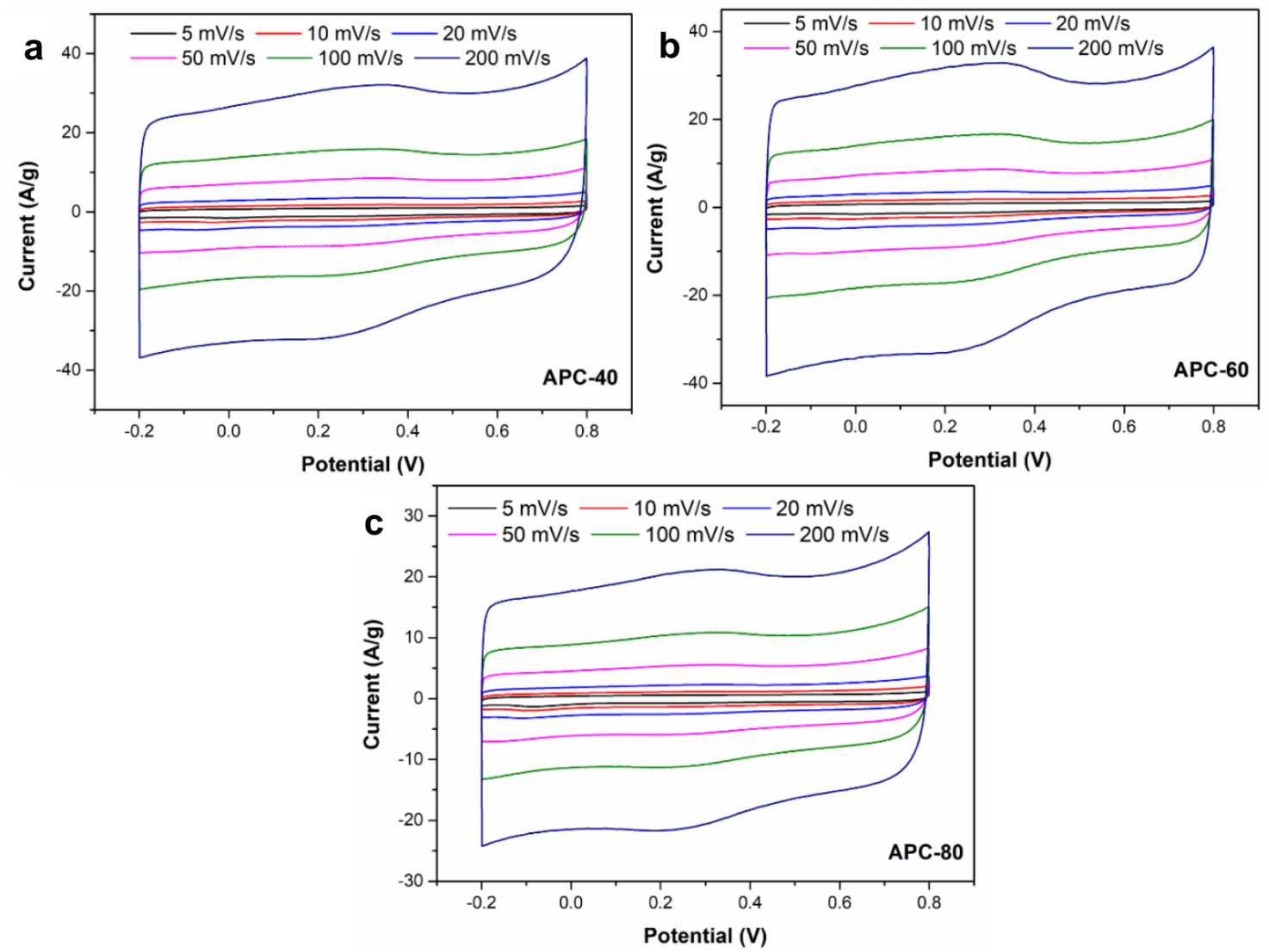

Fig. 5. CV curves of (a) APC-40, (b) APC-60, and (c) APC-80 at different scan rates

Comparing the $\mathrm{CV}$ curves of three samples at the same scan rate, according to Eq. 1 , it is evident that the specific capacitance of APC-60 was higher than that of samples APC-40 and APC-80. The main reasons that APC-60 was higher were the increase of the beating degree, the increase in reaction contact sites of the activator on the surface of flax 
pulp fibers, and the increase in $\mathrm{N}$ and $\mathrm{O}$ elements doped on the surface of the as-prepared APCs, enhancing their surface reactivity and the wettability to the electrolyte. Therefore, the specific capacitance of APC-60 was higher than APC-40. Additionally, the specific capacitance of APC-80 was reduced, mainly because the excessive refining severely damaged the crystallinity of flax pulp fibers, and the carbon skeleton had been more severely damaged during the activation process, resulting in a lower degree of graphitization and poor conductivity of the carbon material. In addition, when the scan rate was increased from $5 \mathrm{mV} / \mathrm{s}$ to $200 \mathrm{mV} / \mathrm{s}$, APC-60 still achieved a high capacitance retention rate of $74.99 \%$, indicating an excellent rate-performance.

\section{Galvanostatic charge-discharge test}

The GCD test of APCs was conducted in the potential window of -0.2 to $0.8 \mathrm{~V}$, and the GCD curves of all the as-prepared APCs working electrodes at different current densities are shown in Figs. 6a through 6c. The GCD curves of APC-40 and APC-60 were linear and almost symmetrical isosceles triangle shapes, demonstrating the exceptional electrochemical reversibility and high columbic efficiency of APC-40 and APC-60. In addition, the curve of APC-80 at $-0.108 \mathrm{eV}$ started to become slightly nonlinear, mainly due to the pseudo-capacitance generated by the redox reaction.
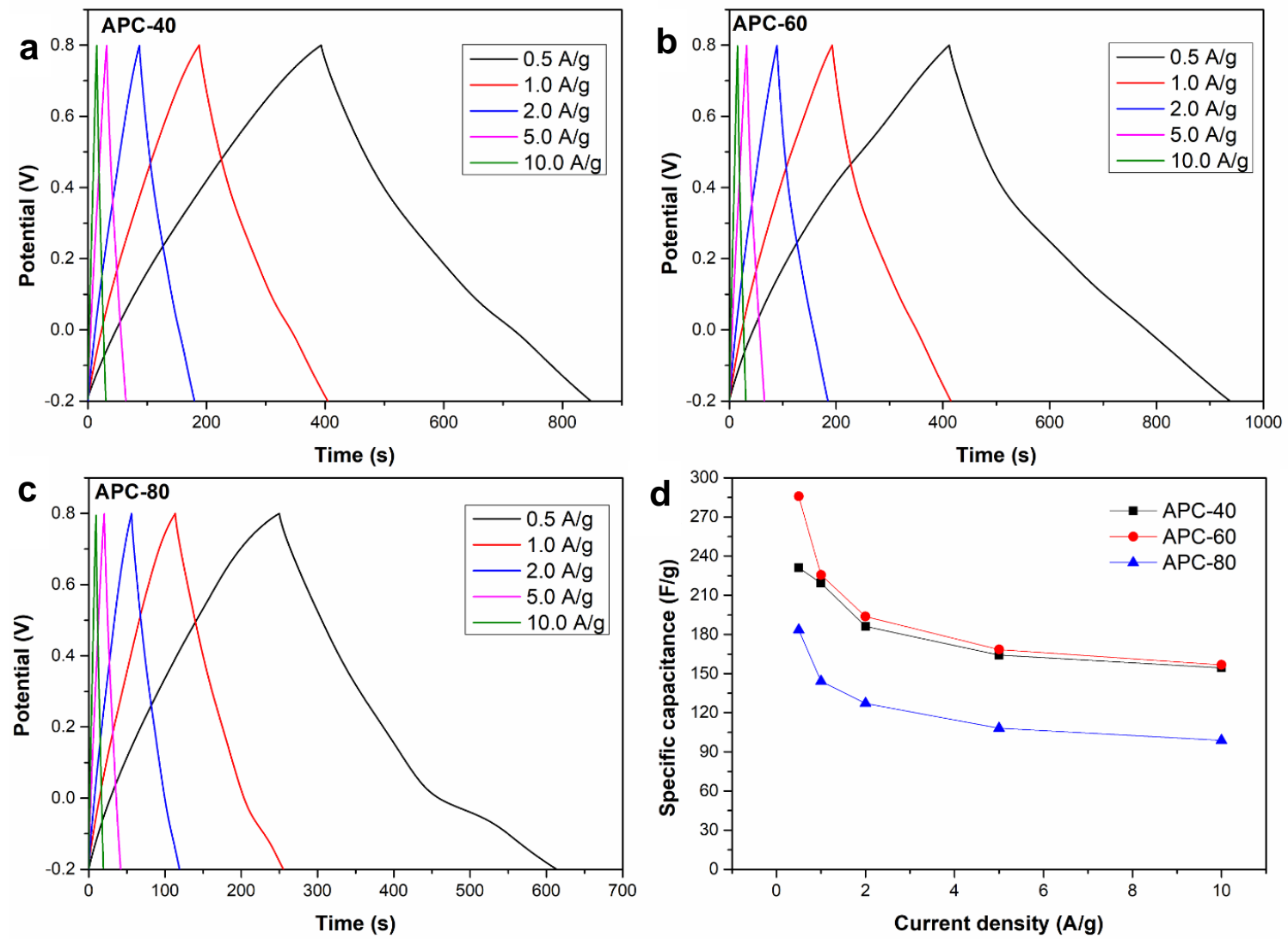

Fig. 6. GCD curves of (a) APC-40, (b) APC-60, (c) APC-80, and (d) specific capacitance of APCs at different current density

According to the calculation of the GCD curve, the specific capacitance of all the samples at different densities are shown in Fig. 6d. When the current density was $0.5 \mathrm{~A} / \mathrm{g}$, the specific capacitances of APC-40, APC-60, and APC-80 were 231.2, 265.8, and 183.5 
F/g, respectively. The specific capacitance of APC-60 was much higher than APC-40 and APC-80, which agreed with the results mentioned in the CV test. A certain degree of refining improved the electrochemical performance of APCs. However, excessive refining reduced the graphitization degree and the conductive performance of the carbon material, causing a poor electrochemical performance. Furthermore, the specific capacitance retention at 59\% remained for the APC-60 electrodes at a high current density of $10 \mathrm{~A} / \mathrm{g}$, indicating that the electrolyte ions quickly and effectively penetrated into the carbon material network structure, and the ions on the electrode/electrolyte interface effectively diffused, providing a large amount of electrolyte ions adsorption under high current density.

\section{Electrochemical impedance spectroscopy analysis}

In order to further evaluate the electrochemical performance of the flax-based APCs, the electrochemical impedance spectroscopy (EIS) of the three-electrode system at open circuit potential was measured. The Nyquist diagrams of APC-40, APC-60, and APC80 are shown in Fig. 7. In the low frequency region, all samples display steep capacitance peaks close to $90^{\circ}$, showing nearly ideal capacitance characteristics. The semicircle shape in the high frequency region represented the charge transfer resistance $\left(R_{\mathrm{ct}}\right)$. However, both APC-40 and APC-60 displayed an unclear semicircle shape, indicating the $R_{\text {ct }}$ of these two samples was small, and the electrolyte ions quickly diffused toward the electrode/ electrolyte interface. In contrast, APC-80 showed a slightly semicircular shape in the high frequency region, indicating that excessive refining caused a poor electrochemical performance of the flax-based porous carbon. The intersection of the Nyquist diagram and the real axis ( $\left.\mathrm{Z}^{\prime}\right)$ was approximately the equivalent series resistance (ESR) of the asprepared electrodes. The ESR of APC-40, APC-60, and APC-80 were 6.74, 7.95, and 8.06 $\Omega$, respectively. It can be seen that as the beating degree increased, the conductivity of the porous carbon material slightly decreased, mainly because the refining severely damaged the crystalline structure of the flax pulp fibers. This damage caused a slightly lower degree of graphitization and a poor conductivity of APCs.

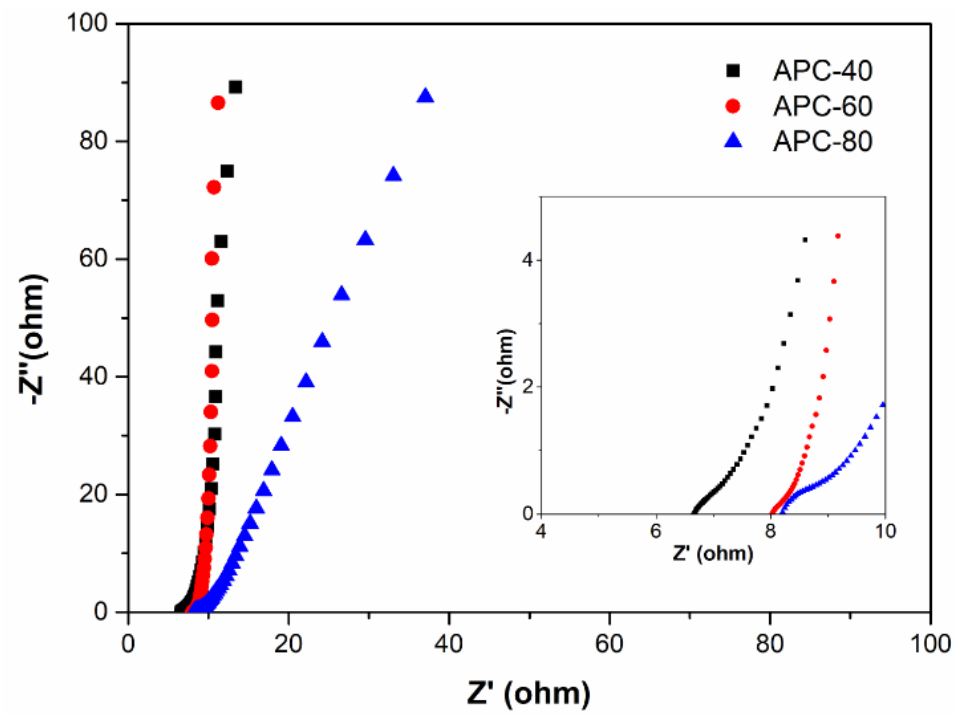

Fig. 7. Nyquist plots of APC-40, APC-60, and APC-80 


\section{CONCLUSIONS}

1. Flax-based activated porous carbons were generated via $\mathrm{KOH}$ and urea synergistic activation in a carbonization process using refined flax pulp as a precursor. With an increased beating degree, the swelling properties of flax pulp fibers improved, and the specific surface area and pore volume of carbon materials gradually increased. APC80 exhibited the highest specific surface area and pore volume, $1255 \mathrm{~m}^{2} / \mathrm{g}$ and 0.724 $\mathrm{cm}^{3} / \mathrm{g}$, respectively.

2. According to XRD and Raman analysis, it was determined that the APCs belonged to amorphous carbon and exhibited a certain graphitization tendency. When the beating degree increased, the degree of graphitization of APCs decreased, and the conductivity of the porous carbon material decreased slightly.

3. Elemental analysis and XPS analysis indicated that APCs were doped with a higher amount of $\mathrm{O}$ element and a small amount of $\mathrm{N}$ element. With the increase of beating degree, O1s on the surface of porous carbon materials increased from $9.19 \%$ to $17.53 \%$, and N1s increased from $0.43 \%$ to $1.15 \%$.

4. According to the electrochemical performance analysis, the flax-based APCsdesigned as a sustainable electrode material — showed excellent rate-performance and electrochemical reversibility. With increased beating degree, the specific capacitance of APCs first increased and then decreased. The APC-60 exhibited the highest specific capacitance of $265.8 \mathrm{~F} / \mathrm{g}$ at a current density of $0.5 \mathrm{~A} / \mathrm{g}$, and the specific capacitance retention at 59\% remained for the APC-60 electrodes at a high current density of 10 $\mathrm{A} / \mathrm{g}$. Thus, it was concluded that the APC-60 could be a promising carbon-based electrode material for preparing supercapacitors with excellent electrochemical performance.

\section{ACKNOWLEDGMENTS}

The authors are grateful for the support from the Natural Science Foundation of Guangdong Province, China.

\section{REFERENCES CITED}

Borenstein, A., Hanna, O., Attias, R., Luski, S., Brousse, T., and Aurbach, D. (2017). "Carbon-based composite materials for supercapacitor electrodes: A review," Journal of Materials Chemistry A 5(25), 12653-12672. DOI: 10.1039/C7TA00863E

Chen, T., Xie, Y., Wei, Q., Wang, X., Hagman, O., Karlsson, O., and Liu, J. (2016). "Effect of refining on physical properties and paper strength of Pinus massoniana and China fir cellulose fibers," BioResources 11(3), 7839-7848. DOI: 10.15376/biores.11.3.7839-7848

Chen, Z., Peng, X., Zhang, X., Jing, S., Zhong, L., and Sun, R. (2017). "Facile synthesis of cellulose-based carbon with tunable $\mathrm{N}$ content for potential supercapacitor application," Carbohydrate Polymers 170, 107-116. DOI: 10.1016/j.carbpol.2017.04.063 
Daems, N., Sheng, X., Vankelecom, I. F. J., and Pescarmona, P. P. (2014). "Metal-free doped carbon materials as electrocatalysts for the oxygen reduction reaction," Journal of Materials Chemistry A 2(12), 4085-4110. DOI: 10.1039/c3ta14043a

Deng, H., Li, G., Yang, H., Tang, J., and Tang, J. (2010). "Preparation of activated carbons from cotton stalk by microwave assisted $\mathrm{KOH}$ and $\mathrm{K}_{2} \mathrm{CO}_{3}$ activation," Chemical Engineering Journal 163(3), 373-381. DOI: 10.1016/j.cej.2010.08.019

Dhakal, H. N., Zhang, Z. Y., Guthrie, R., MacMullen, J., and Bennett, N. (2013). "Development of flax/carbon fibre hybrid composites for enhanced properties," Carbohydrate Polymers 96, 1-8. DOI: 10.1016/j.carbpol.2013.03.074

Fan, Y., Yang, X., Zhu, B., Liu, P. F., and Lu, H. T. (2014). "Micro-mesoporous carbon spheres derived from carrageenan as electrode material for supercapacitors," Journal of Power Sources 268, 584-590. DOI: 10.1016/j.jpowsour.2014.06.100

Feng, H., Zheng, M., Dong, H., Xiao, Y., Hu, H., Sun, Z., Long, C., Cai, Y., Zhao, X., and Zhang, H., et al. (2015). "Three-dimensional honeycomb-like hierarchically structured carbon for high-performance supercapacitors derived from high-ashcontent sewage sludge," Journal of Materials Chemistry A 3(29), 15225-15234. DOI: $10.1039 / \mathrm{c} 5 \mathrm{ta03217b}$

Gharehkhani, S., Sadeghinezhad, E., Kazi, S. N., Yarmand, H., Badarudin, A., Safaei, M. R., and Zubir, M. N. M. (2015). "Basic effects of pulp refining on fiber properties-A review," Carbohydrate Polymers 115, 785-803. DOI: 10.1016/j.carbpol.2014.08.047

Girgis, B. S., Attia, A. A., and Fathy, N. A. (2007). "Modification in adsorption characteristics of activated carbon produced by $\mathrm{H}_{3} \mathrm{PO}_{4}$ under flowing gases," Colloids \& Surfaces A: Physicochemical \& Engineering Aspects 299(1-3), 79-87. DOI: 10.1016/j.colsurfa.2006.11.024

Hadi, P., Xu, M., Ning, C., Lin, C. S. K., and Mckay, G. (2015). "A critical review on preparation, characterization and utilization of sludge-derived activated carbons for wastewater treatment," Chemical Engineering Journal 260, 895-906. DOI: 10.1016/j.cej.2014.08.088

He, D., Wu, L., Yao, Y., Zhang, J., Huang, Z. H., and Wang, M. X. (2020). “A facile route to high nitrogen-containing porous carbon fiber sheets from biomass-flax for high-performance flexible supercapacitors," Applied Surface Science 507, Article ID 145108. DOI: 10.1016/j.apsusc.2019.145108

Islam, M. A., Ahmed, M. J., Khanday, W. A., Asif, M., and Hameed, B. H. (2017). "Mesoporous activated carbon prepared from $\mathrm{NaOH}$ activation of rattan (Lacosperma secundiflorum) hydrochar for methylene blue removal," Ecotoxicology \& Environmental Safety 138, 279-285. DOI: 10.1016/j.ecoenv.2017.01.010

Li, X. F., Chen, W. T., and Dou, L. Q. (2017). "Activated carbon prepared from Alternanthera philoxeroides biomass by one-step $\mathrm{K}_{2} \mathrm{CO}_{3}$ activation," BioResources 12(2), 3340-3350. DOI: 10.15376/biores.12.2.3340-3350

Liang, Y., Li, Y., Wang, H., and Dai, H. (2013). "Strongly coupled inorganic/nanocarbon hybrid materials for advanced electrocatalysis," Journal of the American Chemical Society 135(6), 2013-2036. DOI: 10.1021/ja3089923

Ma, F., Ding, S., Ren, H., and Liu, Y. (2019). "Sakura-based activated carbon preparation and its performance in supercapacitor applications," RSC Advances 9(5), 2474-2483. DOI: $10.1039 / \mathrm{c} 8 \mathrm{ra09685f}$

Mao, J., Heck, B., Abushammala, H., Reiter, G., and Laborie, M. P. (2019). “A structural fibrillation parameter from small angle X-ray scattering to quantify pulp refining," Cellulose 26(7), 4265-4277. DOI: 10.1007/s10570-019-02386-0 
Marrakchi, Z., Khiari, R., Oueslati, H., Mauret, E., and Mhenni, F. (2011). "Pulping and papermaking properties of Tunisian Alfa stems (Stipa tenacissima)-Effects of refining process," Industrial Crops \& Products 34(3), 1572-1582. DOI: 10.1016/j.indcrop.2011.05.022

Martin, N., Davies, P., and Baley, C. (2016). "Evaluation of the potential of three nonwoven flax fiber reinforcements: Spunlaced, needlepunched and paper process mats," Industrial Crops \& Products 83, 194-205. DOI: 10.1016/j.indcrop.2015.10.008

Mohammed, A. A., Chen, C., and Zhu, Z. (2019). "Low-cost, high-performance supercapacitor based on activated carbon electrode materials derived from baobab fruit shells," Journal of Colloid \& Interface Science 538, 308-319. DOI: 10.1016/j.jcis.2018.11.103

Motamedian, H. R., Halilovic, A. E., and Kulachenko, A. (2019). "Mechanisms of strength and stiffness improvement of paper after PFI refining with a focus on the effect of fines," Cellulose 26(6), 4099-4124. DOI: 10.1007/s10570-019-02349-5

Sain, M., and Fortier, D. (2002). "Flax shives refining, chemical modification and hydrophobisation for paper production," Industrial Crops \& Products 15(1), 1-13. DOI: 10.1016/S0926-6690(01)00090-5

Shen, J., Li, J., Qian, X., Ren, W., and Fatehi, P. (2014). “A review on engineering of cellulosic cigarette paper to reduce carbon monoxide delivery of cigarettes," Carbohydrate Polymers 101, 769-775. DOI: 10.1016/j.carbpol.2013.09.101

Sui, Z. Y., Meng, Q. H., Li, J. T., Zhu, J. H., Cui, Y., and Han, B. H. (2014). "High surface area porous carbons produced by steam activation of graphene aerogels," Journal of Materials Chemistry A 2(25), 9891-9898. DOI: 10.1039/c4ta01387e

Tan, X. F., Liu, S. B., Liu, Y. G., Gu, Y. L., Zeng, G. M., Hu, X. J., Wang, X., Liu, S. H., and Jiang, L. H. (2017). "Biochar as potential sustainable precursors for activated carbon production: Multiple applications in environmental protection and energy storage," Bioresource Technology 227, 359-372. DOI: 10.1016/j.biortech.2016.12.083

Tang, L., Feng, Y., He, W., and Yang, F. (2019). "Combination of graphene oxide with flax-derived cellulose dissolved in $\mathrm{NaOH} /$ urea medium to generate hierarchically structured composite carbon aerogels," Industrial Crops \& Products 130, 179-183. DOI: $10.1016 /$ j.indcrop.2018.12.068

Wang, J., and Kaskel, S. (2012). "KOH activation of carbon-based materials for energy storage," Journal of Materials Chemistry 22(45), 23710-23725. DOI: 10.1039/c2jm34066f

Wang, K., Zhao, N., Lei, S., Yan, R., Tian, X., Wang, J., Song, Y., Xu, D., Guo, Q., and Liu, L. (2015). "Promising biomass-based activated carbons derived from willow catkins for high performance supercapacitors," Electrochimica Acta 166, 1-11. DOI: 10.1016/j.electacta.2015.03.048

Wu, F., Gao, J., Zhai, X., Xie, M., Sun, Y., Kang, H., Tian, Q., and Qiu, H. (2019). "Hierarchical porous carbon microrods derived from albizia flowers for high performance supercapacitors," Carbon 147, 242-251. DOI:

10.1016/j.carbon.2019.02.072

Wu, H., Cheng, L., Zhang, Y., Yuan, W., Zheng, L., and Yuan, X. (2017). "Free-standing activated flax fabrics with tunable meso/micropore ratio for high-rate capacitance," Carbon 116, 518-527. DOI: 10.1016/j.carbon.2017.02.029 
Yan, L., Chouw, N., and Jayaraman, K. (2014). "Flax fibre and its composites-A review," Composites Part B: Engineering 56, 296-317. DOI: 10.1016/j.compositesb.2013.08.014

Yang, J., and Qiu, K. (2010). "Preparation of activated carbons from walnut shells via vacuum chemical activation and their application for methylene blue removal," Chemical Engineering Journal 165(1), 209-217. DOI: 10.1016/j.cej.2010.09.019

Yang, X., Fei, B., Ma, J., Liu, X., Yang, S., Tian, G., and Jiang, Z. (2018). "Porous nanoplatelets wrapped carbon aerogels by pyrolysis of regenerated bamboo cellulose aerogels as supercapacitor electrodes," Carbohydrate Polymers 180, 385-392. DOI: 10.1016/j.carbpol.2017.10.013

Yu, X., Wang, S., and Zhang, J. (2017). "Preparation of high adsorption performance activated carbon by pyrolysis of waste polyester fabric," Journal of Materials Science 53(7), 5458-5466. DOI: 10.1007/s10853-017-1928-2

Yu, Z., Tetard, L., Zhai, L., and Thomas, J. (2015). "Supercapacitor electrode materials: Nanostructures from 0 to 3 dimensions," Energy \& Environmental Science 8(3), 702730. DOI: $10.1039 / \mathrm{c} 4 \mathrm{ee} 03229 \mathrm{~b}$

Zhang, L. L., and Zhao, X. S. (2009). "Carbon-based materials as supercapacitor electrodes,” Chemical Society Reviews 38(9), 2520-2531. DOI: 10.1039/b813846j

Article submitted: September 25, 2020; Peer review completed: November 15, 2020; Revised version received: December 17, 2020; Accepted: December 18, 2020; Published: December 23, 2020.

DOI: $10.15376 /$ biores.16.1.1296-1310 